\title{
Non-linear and Non-planar Free Thermal Vibration of SWNT in Molecular Dynamic Simulation
}

\author{
Heeyuen Koh, James Cannon, Shohei Chiashi, Junichiro Shiomi and Shigeo Maruyama \\ Department of Mechanical Engineering, The University of Tokyo \\ 7-3-1 Hongo, Bunkyo-ku, Tokyo 113-8656, Japan \\ Phone: +81-3-5841-6421 E-mail: maruyama@photon.t.u-tokyo.ac.jp
}

\section{Introduction}

Vibration of suspended and cantilevered nanotubes and nanowires has been studied extensively in electronics circuits such as transistor, mixer[1], sensors[2-3] and mesoscopic quantum theory[4].

Recently, it is reported that the power spectrum of the free thermal vibration of cantilevered single walled carbon nanotubes (SWNT) can be well expressed by Euler beam and Timoshenko beam theory and the continuous mechanics portion of the motion among the total thermal energy is about 97 percent [5-6].

The beam motion and thermal vibrational motion are strongly coupled for nanoscale structures. Vibrational motion or temperature can evolve to the beam motion. We observed that there is nonplanar motion with beating and bifurcation with long time span in NVE condition using molecular dynamics (MD) calculation. A few experiments are reported about free thermal vibration of the nanoscale cantilevered and suspended structure. Non planar motion of free vibration[7] and forced vibration[8] is measured using the field emission method (FEM) in silicon nanowire.

We show that poincare distribution and frequency response in fine resolution shows this doffing (nonplanar) motion satisfies the solution of continuum non-linear equation which is derived by Hamilton's extended principle[9].

\section{Theory}

We extended non-planar non-linear continuum theory which has once reported to explain the forced nonlinear motion of suspended SWNT in field effective transistor (FET) system[10]. In continuum dynamics, the main reasons of nonplanar and non-linear motion of 1D structure are supposed to be the longitudinal extensibility of structure and torsional motion.

In our theory, extensibility is considered. From the extended form of Hamiltonian which has the strain energy over this assumption, we can derive the non-dimensionalized nonlinear equation for cantilever beam motion as followings:

$$
\begin{aligned}
& \hat{u}_{, t t}+\frac{I_{y y}}{A L^{2}} \hat{u}_{, s s s s}-\frac{1}{2} \hat{u}_{, s s} \int_{0}^{1}\left[\left(\hat{u}_{, s}\right)^{2}+\left(\hat{v}_{, s}\right)^{2}+\varepsilon\right] d s=0,(1) \\
& \hat{v}_{, t t}+\frac{I_{y y}}{A L^{2}} \hat{v}_{, s s s s}-\frac{1}{2} \hat{v}_{, s s} \int_{0}^{1}\left[\left(\hat{u}_{, s}\right)^{2}+\left(\hat{v}_{, s}\right)^{2}+\varepsilon\right] d s=0
\end{aligned}
$$

$$
\left[w_{, z}+\frac{1}{2}\left(u_{, z}\right)^{2}+\frac{1}{2}\left(v_{, z}\right)^{2}\right]_{, z}=0,
$$

here, $\hat{u}, \hat{v}$ and $\widehat{w}$ are non dimensional displacements along $\mathrm{x}, \mathrm{y}$ and $\mathrm{z}$ directions. Tube axis is aligned along $\mathrm{z}$ axis. $A$ is effective area of tube, $L$ is tube length, $\mathrm{s}$ is the non dimensionalized variable of $\mathrm{z}(s=z / L)$ and $\varepsilon$ is longitudinal strain. The first two terms in Eq. (1) and (2) are classic beam equation and last integration comes from the extensibility assumption. Eq. (3) is the equation for longitudinal displacement condition. The kinetic energy along the tube axis is ignored.

The analytical solutions for Eq. (1) and (2) are integrated using Galerkin method and KGM method[9] like:

$$
\begin{gathered}
\hat{u}=a(\tau) \cos (\omega \tau+\theta)+\varepsilon g, \\
\hat{v}=b(\tau) \sin (\omega \tau+\theta)+\varepsilon h,
\end{gathered}
$$

where $a(\tau)$ and $b(\tau)$ satisfies:

$$
\begin{gathered}
a^{2}(\tau)+b^{2}(\tau)=c_{1}^{2}, \\
a^{2}(\tau) b^{2}(\tau)=c_{2}^{2} \sec ^{2}(\varphi-\theta),
\end{gathered}
$$

$a^{2}(\tau)$ or $b^{2}(\tau)=$

$$
\frac{c_{1}^{2}}{2} \pm \sqrt{\left(\frac{c_{1}^{2}}{2}\right)^{2}-c_{2}^{2}} \sin \left[\frac{\varepsilon q c_{2}}{2 \omega} \tau+c_{3}\right]
$$

$$
\begin{aligned}
& \theta \text { or } \varphi=\frac{3 \varepsilon}{8 \omega} q c_{1}^{2} \tau \\
& \quad-\tan ^{-1}\left\{\frac{\mathrm{c}_{1}^{2} \tan \left[\left(\frac{\varepsilon q c_{2}}{4 \omega}\right) \tau+c_{3} / 2\right] \pm \sqrt{\mathrm{c}_{1}^{4}-4 \mathrm{c}_{2}^{2}}}{2 \mathrm{c}_{2}}\right\}
\end{aligned}
$$

Double signs are used with same order. Integration constants $c_{1}$ through $c_{6}$ are obtained using energy conservation condition and initial conditions. This constant is dependent on the temperature and vibration frequency.

\section{Simulation}

The simulation using $8 \mathrm{~nm}$ and $10 \mathrm{~nm}$ long $(5,5)$ and $(10,10)$ SWNT is performed with long time span to consider the stability of motion. Unit cell includes 10 and 20 atoms respectively. Bond length is $\mathrm{a}_{\mathrm{c}-\mathrm{c}}=0.142$ 

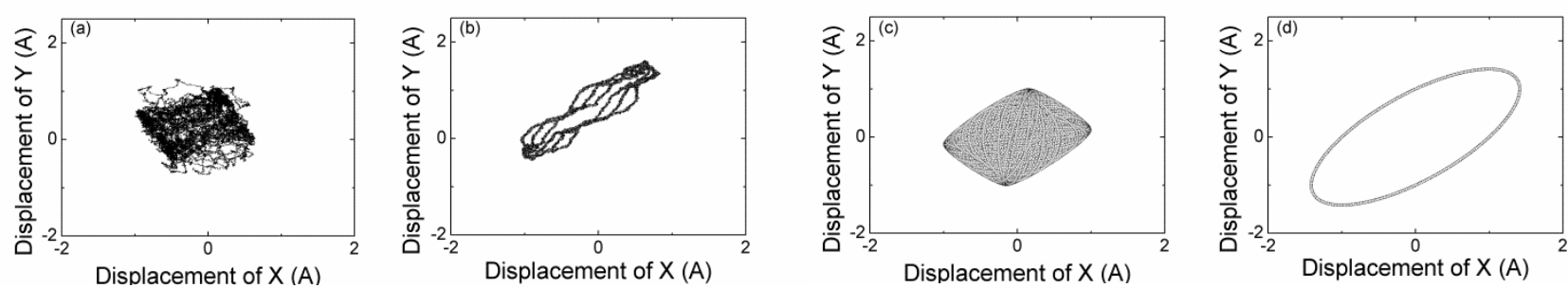

Figure 1. Poincare map of the cantilevered $8 \mathrm{~nm}$ and $25 \mathrm{~nm}$ long $(5,5) \mathrm{SWNT}$ at $50 \mathrm{~K}$. The trajectory is come from the tip location. (a) MD calculation with $8 \mathrm{~nm}$ length SWNT, (b) MD calculation with $25 \mathrm{~nm}$, (c) Nonlinear Continuum theory with $8 \mathrm{~nm}$ SWNT (d)

Nonlinear Continuity theory with $25 \mathrm{~nm}$.

$\mathrm{nm}$ and the LAMMPS package code was used with Brenner potential function which is tuned for phonon dispersion. Each condition is initially set at $50 \mathrm{~K}$ and langevin thermostat was used with the damping coefficient 0.01. Time step size is 0.5 fs. After initialization, it is calculated in NVE with fixed boundary.

The temperature profile in time domain supports constant energy state. However, unlike the temperature and total energy, the displacement profiles show beating and whirling motion. To observe this, the tip displacement and velocity was averaged from the ring which is located at $7.5 \mathrm{~nm}$ and $22.8 \mathrm{~nm}$ from the bottom.

\section{Result and discussion}

Figure 1 shows that Poincare maps calculated from MD and continuum theory. They show similar overall trajectories for both length. In case of 8nm long SWNT, Poincare map is the duplication of the trajectories of several cycles which has different path. These trajectories for each cycle and its order also can be explained from the analytical solution of Eq. (1) and Eq (2). Since this solution has time varying amplitude, beating or couple of more frequency near the $1^{\text {st }}$ mode is very natural in nonlinear theories.

Based on the similarity of MD calculation and nonlinear continuum theory in Poincare map, we can confirm the assumption of the longitudinal displacement and Green strain. According to the derivation, it is impossible to have the in-plane bending motion in free standing condition with the displacement along tube axis. So far, this non linear motion equation tells us that the size of tubes which decides the frequency in non dimensionalized motion and the temperature which influences the amplitude of displacement in lateral and axial direction is the determining parameter for duffing.

The reason why it follows continuum scale dynamic strain energy is yet not clear. For certain, this nonlinear motion should be consisting of its eigenmodes of the structure and their interaction. In nanoscale, it could mean this periodic non linear motion is due to the aggregation of phonons. Developing the more concrete analysis will be meaningful for various multiscale and multi physics calculations to deal with cantilevered and suspended nano structure.

\section{Summary}

We observed the non-planar duffing motion in free thermal vibrations of SWNT and found that this motion can be expressed using non linear continuum dynamics equation. We derived the analytic solution from the extended form of Hamiltonian and KGM method.

\section{Acknowledgement}

Part of this work was financially supported by Grant-in-Aid for Scientific Research (19054003 and 22226006), and Global COE Program 'Global Center for Excellence for Mechanical Systems Innovation'.

\section{Reference}

[1] V. Sazonova, Y. Yaish, H. Üstüne, D. Roundy, T. A. Arias \& Paul L. McEuen, Nature, 431, 284 (2004)

[2] H.-Y. Chiu, P. Hung, H. W. Ch. Postma and M. Bockrath, Nano Lett., 8, 4342 (2008)

[3] C. Stampfer, A. Jungen, R. Linderman, D. Obergfell, S. Roth, and C. Hierold, Nano Lett., 6, 1449 (2006)

[4] S.M. Carr, W.E. Lawrence, M.N. Wybourne, Phy. Rev. B, 316-317, 464 (2002)

[5] E. H. Feng and R. E. Jones, Phy. Rev. B, 81, 125436 (2010)

[6] E. H. Feng and R. E. Jones, Phy. Rev. B, 83, 195412 (2011)

[7]A. Ayari, P. Vincent, S. Perisanu, M. Choueib, V. Gouttenoire, M. Bechelany, D. Cornu, and S. T. Purcell, Nano Lett., 7, 2252 (2007)

[8] S. Perisanu, T. Barois, A. Ayari, P. Poncharal, M. Choueib, S. T. Purcell, and P. Vincent, Phy. Rev. B, 81, 165440 (2010)

[9] C.-H. Ho, R.A. Scott and J.G. Eisley, Joun. Soun. Vib., 47, 333 (1976)

[10]W. G. Conley, A. Raman, C. M. Krousgrill, and S. Mohammadi, Nano Lett., 8, 1590-1595 